\title{
The Phase Diagrams of Spin-1/2 Ising Model on a Two-Layer Bethe Lattice with AFM/AFM Interactions
}

\author{
E. Albayrak And A. Yigit \\ Department of Physics, Erciyes University, 38039, Kayseri, Turkey
}

(Received November 18, 2008; in final form May 14, 2009)

\begin{abstract}
The phase diagrams of spin-1/2 Ising model on a two-layer Bethe lattice with antiferromagnetic interactions for each layer and either antiferromagnetic or ferromagnetic interaction between the layers are investigated by using the pairwise approach for given values of coordination number $q$. The exact expressions of the order-parameters, response functions and free energy are obtained in terms of the recursion relations. The ground-state phase diagrams are calculated for given system parameters of the model. In the guidance of the ground-state phase diagrams, the temperature dependent phase diagrams of the model are also studied in detail for given coordination numbers $q=3,4$ and 6 . It was found that the system presents only second-order phase transitions with different thermal behaviors for all values of $q$. In addition, two Néel temperatures, $T_{\mathrm{N}}$, are found for $q=6$ only.
\end{abstract}

PACS numbers: 05.50.+q, 68.35.Rh, 64.60.Cn

\section{Introduction}

In the recent years, the ferromagnetic (FM) or antiferromagnetic (AFM) materials or their combinations were simulated as multi-layered systems to simulate superlattices, thin films etc. and their magnetic behaviors were investigated with many respects. The Ising or Heisenberg spins were considered in modeling of these structures.

The symmetric two-layer Ising model was studied by the corner transfer matrix renormalization group method to calculate the critical points and critical exponents [1]. The Monte Carlo (MC) simulation was used to study the behavior of an Ising model consisting of two FM layers with different interaction constants coupled weakly together [2]. The role of the interlayer coupling between $\mathrm{CuO}_{2}$ planes on bilayer-group high- $T_{\mathrm{c}}$ superconductors was studied within a simple randomly decorated bilayer Ising model and a Bethe-lattice approach [3]. A two-layer Ising system was studied by means of a mean field theory (MFT), a generalized MFT, a scaling approach and high-temperature series expansions [4]. The critical temperature for layered Ising model on the sc lattice was calculated using the transfer-matrix version of a MF approximation [5]. The critical temperature in the two-layered Ising model was precisely calculated by using the transfer matrix MF approximation [6]. The two-layered spin-3/2 Ising model was studied by using the interfacial approximation for a system with competing antiferromagnetic and ferromagnetic interactions on a square lattice [7]. The effect of a variable surface transverse magnetic field on the order-disorder layering transitions of an Ising model was investigated using the MFT and finite cluster approximation [8]. The multilayer spin- $1 / 2$ square lattice Heisenberg antiferromagnet with up to six layers was studied using series expansions [9]. A simple criterion which allows us for the determination of the order-disorder critical temperatures was found and predicted that $\beta_{\mathrm{c}}=0.2656$ for the two coupled layers of Ising spins [10]. The MC simulations were used to study the phase transitions on coupled anisotropic FM/AFM films with classical Heisenberg spins [11]. A variety of the aspects of a bilayer system of Ising spins was studied including accurate estimates of the critical temperature for FM interactions, scaling of the critical temperature when the interlayer interaction goes to zero, and approximations of the phase diagrams for the case when AFM interlayer interactions are present including location of the tricritical point [12]. A magnetic impurity in two different $S=1 / 2$ Heisenberg bilayer antiferromagnets at their respective critical interlayer couplings separating Néel and disordered ground states (GSs) was considered and the impurity susceptibility was calculated by using a quantum MC method [13]. The quantum MC data was analyzed in the vicinity of the quantum transition between a Néel state and a quantum paramagnet in a two-layer, square spin-1/2 Heisenberg antiferromagnet [14]. A two-layer Heisenberg antiferromagnet which can be either in the Néel-ordered or in the disordered phase at $T=0$, depending on the ratio of the intra-layer and inter-layer exchange constants, was investigated [15]. The correlation length and the static structure factor for bilayer antiferromagnets, such as $\mathrm{YBa}_{2} \mathrm{Cu}_{3} \mathrm{O}_{6}$, were calculated using field theoretical and numerical methods [16]. High-temperature, Ising and dimer expansions were de- 
veloped for studying the two-layer spin- $1 / 2$ square-lattice Heisenberg antiferromagnet [17]. The antiferromagnetic order-disorder transition occurring at $T=0$ in a two-layer quantum Heisenberg antiferromagnet was studied as the interplane coupling was increased [18].

The similar approaches are also employed for the examination of the superlattices; a magnetic superlattice was examined within the framework of the the effective field theory (EFT) with correlations for arbitrary number of layers [19], the magnetic superlattice consisting of two different FM was examined in a transverse Ising model using the EFT that accounts for the self-spin correlation function [20], infinite $\mathrm{FM} \mathrm{AB} \mathrm{AB}_{2}$-superlattice and $\mathrm{A}\left(n_{a}\right) \mathrm{B}\left(n_{b}\right)$ sandwich structures, with constituents composed of various ferromagnets with equal thickness, were investigated using an Ising model in a linear cluster approximation [21]. The fcc superlattices of equal-thickness $\mathrm{Ni}$ and Fe layers were modeled with a short range interaction Ising model using parameters determined from a previous study of homogeneous $\mathrm{Ni}_{c} \mathrm{Fe}_{1-c}$ alloys [22] and an Ising model was presented for a simple cubic-type structure to determine the magnetic properties for superlattices of periodic $\mathrm{A}_{k}\left(\mathrm{~A}_{p} \mathrm{~B}_{1-p}\right) 1 \mathrm{~B}_{h}$ formula consisting of $k$ layers of spin- $1 / 2 \mathrm{~A}$ ions, $h$ layers of spin- $1 / 2 \mathrm{~B}$ ions and a single layer disordered alloy interfaces between them [23].

It should be mentioned that the two-layer Ising models are also used to simulate thin films: The elementary excitations for a uniaxial AFM film with an external magnetic field $H$ applied along the easy axes was studied by using a microscopic approach [24]. A vectorized multispin-coding MC method was used to determine the behavior of the surface-layer magnetization at the bulk transition in a simple cubic Ising film with strongly enhanced surface coupling [25]. Within the framework of the EFT, the phase transitions of an Ising film in a transverse field were investigated [26]. The effect of a random field within the bimodal distribution on the layering transitions in a spin- $1 / 2$ Ising thin film was investigated by using MC simulations [27]. The influence of corrugation and disorder at the surface on the critical behavior of a FM Ising film was investigated using the MFT and the cluster approximation [28]. The critical behavior of both semi-infinite spin- $1 / 2$ and spin- $1 / 2$ Ising film in a random transverse surface field has been studied within the EFT [29]. The FM multilayers consisting of two materials in a sandwich structure (ABA) using an EFT with a probability distribution technique that accounts for the self-correlation function [30]. The finite size transfer matrix method and MFT were used to study the layering transitions for an Ising model under uniform and variable magnetic field in a film of finite thickness limited by a single substrate [31].

Let us note that the fictitious lattices were also employed in studying the multilayered structures. Thus, a two-layer Bethe lattice for an Ising model was studied by using an iteration technique [32], the Ising crossover between the multilayer and single layer Sierpinski gaskets — a fractal lattice — was used to investigate the critical behavior of an Ising model [33] and the behavior of the Ising thin films through the use of layered Bethe lattices and Husimi trees were studied [34].

In this work in order to simulate a two-layer Ising model, two Bethe lattices each with a branching ratio of $q$ Ising spins were placed parallel to each other with an exact match as seen in Fig. 1. The case for the AFM type intralayer and either AFM or FM type interlayer bilinear interactions are assumed. The problem is approached by the use of the exact recursion relations in a pairwise approximation [32] by dividing the two layered Bethe lattice into the sublattices A and B. Thus, the GS phase diagrams (PDs) and the temperature dependent PDs of the model are obtained in detail.

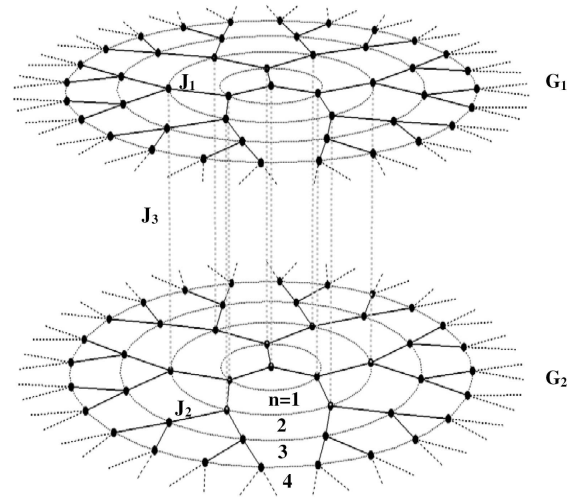

Fig. 1. The two-layer Bethe lattice of coordination number $q=3.0$. $G_{1}$ and $G_{2}$ refer to the upper and lower layers containing the spins labeled as $S_{i}$ and $\sigma_{i^{\prime}}$, respectively.

The rest of the work is set up as follows. In Sect. 2, the two-layer Ising model is introduced and then the formulations of the order-parameters, free energy and the response functions are obtained exactly in terms of the recursion relations. The illustrations and discussions of the PDs are presented in Sect. 3. Finally, the last section is a brief summary and discussions.

\section{The model and formulation}

We consider two identical layers of the Bethe lattices $G_{1}$ and $G_{2}$ which are placed parallel to each other with an exact match forming the two-layer Bethe lattice as seen in Fig. 1. The Ising model is simulated on a two-layer Bethe lattice and a pairwise approach is employed for its investigation. In this approach, an adjacent nearest neighbor (NN) pair is picked from deep inside the two-layer Bethe lattice, called as the central pair, which forms the first-generation spins. This central pair is connected to $q \mathrm{NN}$ spin pairs (i.e. $q$ is the coordination number) which form the second-generation spins. Each pair of the second-generation is joined to $q-1 \mathrm{NNs}$. Therefore, in total it has $q(q-1)$ NNs which form the third-generation and so on to infinity. As a result each spin has $(q+1) \mathrm{NN}$ spins, i.e. $q \mathrm{NNs}$ from its layer and one from the adjacent layer. 
Thus the appropriate Hamiltonian of such a two-layer Bethe lattice model in the existence of external magnetic field may be given as

$$
\begin{aligned}
\mathcal{H} & =-J_{1} \sum_{\langle i j\rangle} S_{i} S_{j}-J_{2} \sum_{\left\langle i^{\prime} j^{\prime}\right\rangle} \sigma_{i^{\prime}} \sigma_{j^{\prime}}-J_{3} \sum_{\left\langle i i^{\prime}\right\rangle} S_{i} \sigma_{i^{\prime}} \\
& -H_{1} \sum_{i} S_{i}-H_{2} \sum_{i^{\prime}} \sigma_{i^{\prime}} .
\end{aligned}
$$

$S_{i}$ and $\sigma_{i^{\prime}}$ take the values $\pm 1 / 2$ and refer to the spins in layers $G_{1}$ and $G_{2}$, respectively. $J_{1}$ and $J_{2}$ are the intralayer bilinear interactions of the $q \mathrm{NN}$ spins of the layers. Thus, the first and second summations are over all NN sites of $G_{1}$ and $G_{2}$, respectively. $J_{3}$ is the interlayer bilinear interaction of adjacent $\mathrm{NN}$ spins between the layers, therefore, the third summation runs over all the adjacent neighboring sites of $G_{1}$ and $G_{2}$. The layers are assumed to be under the influence of external magnetic fields $H_{1}$ and $H_{2}$, thus the fourth and final sums run over all the lattice sites in each layer.

The partition function which is given by definition as

$$
\mathbb{Z}=\sum_{\text {All Config. }} \mathrm{e}^{-\beta \mathcal{H}}=\sum_{\mathrm{Spc}} P(\mathrm{Spc}),
$$

where $P(\mathrm{Spc})$ is the unnormalized probability distribution, $\beta=1 / k T$ is the inverse temperature and $k$ is the Boltzmann constant and the summation runs over all the spin configurations $(\mathrm{Spc})$. Beginning from the central pair on the lattice made up with $q$ separate branches connecting each pair of spins, one follows only one of the branches out of $q$, therefore, for the full formulation we have to define the partition function for each of these separate branches called as $g_{n}(S, \sigma)$. Each spin $S_{i}$ and spin $\sigma_{i^{\prime}}$ can take the values $\pm 1 / 2$, thus we have to define four $g_{n}(S, \sigma)$ functions for $2^{2}=4$ configurations for each pair of spins. As a result in this pairwise approach, 3 exact recursion relations are defined as the ratios of these partition functions of the separate branches as

$$
\begin{aligned}
& X_{n}=\frac{g_{n}(1 / 2,1 / 2)}{g_{n}(-1 / 2,-1 / 2)}, \quad Y_{n}=\frac{g_{n}(1 / 2,-1 / 2)}{g_{n}(-1 / 2,-1 / 2)}, \\
& Z_{n}=\frac{g_{n}(-1 / 2,1 / 2)}{g_{n}(-1 / 2,-1 / 2)} .
\end{aligned}
$$

Each recursion relation is a function of the recursion relations for the NN shell with $n-1$, therefore in order to obtain their numerical values for given system parameters an iteration method may be employed. Meanwhile, the choice of the recursion relations, i.e. the ratios of these $g_{n}(S, \sigma)$ functions, are completely arbitrary. In the thermodynamic limit $(n \rightarrow \infty)$, they determine the states of the system, and thus they may be called as the equations of state.

The magnetizations $M_{1}$ and $M_{2}$ of the layers $G_{1}$ and $G_{2}$ are defined as

$$
M_{1}=\langle S\rangle \text { and } M_{2}=\langle\sigma\rangle,
$$

where $\langle\ldots\rangle$ denotes the usual thermal average. They are calculated in terms of the recursion relations as

$$
M_{1}=\left[\mathrm{e}^{\beta\left(+0.25 J_{3}+0.5 H_{1}+0.5 H_{2}\right)} X_{n}^{q}\right.
$$

$$
\begin{aligned}
& +\mathrm{e}^{\beta\left(-0.25 J_{3}+0.5 H_{1}-0.5 H_{2}\right)} Y_{n}^{q} \\
& -\mathrm{e}^{\beta\left(-0.25 J_{3}-0.5 H_{1}+0.5 H_{2}\right)} Z_{n}^{q} \\
& \left.-\mathrm{e}^{\beta\left(+0.25 J_{3}-0.5 H_{1}-0.5 H_{2}\right)}\right] / 2 \mathbb{Z}
\end{aligned}
$$

and

$$
\begin{gathered}
M_{2}=\left[\mathrm{e}^{\beta\left(+0.25 J_{3}+0.5 H_{1}+0.5 H_{2}\right)} X_{n}^{q}\right. \\
-\mathrm{e}^{\beta\left(-0.25 J_{3}+0.5 H_{1}-0.5 H_{2}\right)} Y_{n}^{q} \\
+\mathrm{e}^{\beta\left(-0.25 J_{3}-0.5 H_{1}+0.5 H_{2}\right)} Z_{n}^{q} \\
\left.-\mathrm{e}^{\beta\left(+0.25 J_{3}-0.5 H_{1}-0.5 H_{2}\right)}\right] / 2 \mathbb{Z},
\end{gathered}
$$

where

$$
\begin{aligned}
\mathbb{Z} & =\mathrm{e}^{\beta\left(0.25 J_{3}+0.5 H_{1}+0.5 H_{2}\right)} X_{n}^{q} \\
& +\mathrm{e}^{\beta\left(-0.25 J_{3}+0.5 H_{1}-0.5 H_{2}\right)} Y_{n}^{q} \\
& +\mathrm{e}^{\beta\left(-0.25 J_{3}-0.5 H_{1}+0.5 H_{2}\right)} Z_{n}^{q} \\
& +\mathrm{e}^{\beta\left(0.25 J_{3}-0.5 H_{1}-0.5 H_{2}\right)} .
\end{aligned}
$$

The two response functions are the susceptibility and the specific heat. The constant magnetic field susceptibilities of the layers $G_{1}$ and $G_{2}$ are given by the definition as

$$
\chi_{1}=\lim _{H_{1} \rightarrow H} \frac{\partial M_{1}}{\partial H_{1}} \quad \text { and } \quad \chi_{2}=\lim _{H_{2} \rightarrow H} \frac{\partial M_{2}}{\partial H_{2}}
$$

and the total susceptibility of the bilayer is just the sum of the susceptibilities of the layers

$$
\chi_{\text {Total }}=\chi_{1}+\chi_{2} \text {. }
$$

In order to obtain the specific heat, the free energy in terms of the recursion relations is needed. Therefore, the free energy of the bilayer Bethe lattice is given as

$$
-\beta F=\frac{q}{2} \log \mathbb{W}+\frac{2-q}{2} \log \mathbb{Z}
$$

and which is used to find the places of the first-order phase transition temperatures, if any exist, and the stable solutions and where

$$
\begin{aligned}
\mathbb{W} & =\mathrm{e}^{\beta\left[0.25\left(-J_{1}-J_{2}+J_{3}\right)+0.5\left(H_{1}+H_{2}\right)\right]} X_{n}^{q-1} \\
& +\mathrm{e}^{\beta\left[0.25\left(-J_{1}+J_{2}-J_{3}\right)+0.5\left(H_{1}-H_{2}\right)\right]} Y_{n}^{q-1} \\
& +\mathrm{e}^{\beta\left[0.25\left(J_{1}-J_{2}-J_{3}\right)+0.5\left(-H_{1}+H_{2}\right)\right]} Z_{n}^{q-1} \\
& +\mathrm{e}^{\beta\left[0.25\left(J_{1}+J_{2}+J_{3}\right)-0.5\left(H_{1}+H_{2}\right)\right]} .
\end{aligned}
$$

The specific heat at constant magnetic fields is defined as

$$
C_{H}=T\left(\frac{\partial S}{\partial T}\right)_{H}=-T\left(\frac{\partial^{2} F}{\partial T^{2}}\right)_{H},
$$

where $H$ refers to $H_{1}$ and $H_{2}$ and $S$ is the entropy. To be consistent with the variables used in this calculations, one can write the specific heat by using a chain rule as

$$
C / k=-\beta^{\prime}\left[2 \beta^{\prime} \frac{\partial\left(F / J_{1}\right)}{\partial \beta^{\prime}}+\beta^{\prime 2} \frac{\partial^{2}\left(F / J_{1}\right)}{\partial \beta^{\prime 2}}\right],
$$

which is not as simple as it is seen, since it contains the first- and second-partial derivatives of the recursion relations with respect to $\beta^{\prime}=\beta J_{1}$, therefore, the explicit equation is too long to be given here. 
So far we have only obtained the formulation for general interactions, but for the AFM interactions for the layers, i.e. $J_{1}<0$ and $J_{2}<0$, we have to partition each layer into sublattices $\mathrm{A}$ and $\mathrm{B}$. Hence, the recursion relations in a pairwise approach for the two-layer in terms of the sublattices A and B may be written as follows:

$$
\left\{X_{n}, Y_{n}, Z_{n}\right\} \rightarrow \begin{array}{ll}
\left\{X_{n}^{\mathrm{A}}, Y_{n}^{\mathrm{A}}, Z_{n}^{\mathrm{A}}\right\} & \text { for even } n, \\
\left\{X_{n}^{\mathrm{B}}, Y_{n}^{\mathrm{B}}, Z_{n}^{\mathrm{B}}\right\} & \text { for odd } n .
\end{array}
$$

The notable point of this approach is that non-staggered phases are described by the single fixed points $\left\{X_{n}, Y_{n}, Z_{n}\right\} \rightarrow\{X, Y, Z\}$, while the staggered phases appear as two-cycle double points as indicated above.

In addition, the sublattice magnetizations for the layers $G_{1}$ and $G_{2}$, since each spin only interacts with its NNs from its own layer and a NN from the adjacent layer with the same sublattice, i.e. A or B, could be written as

$$
\left\{M_{1,2}\right\} \rightarrow \begin{array}{ll}
\left\{M_{1 \mathrm{~A}}, M_{2 \mathrm{~A}}\right\} & \text { for even } n, \\
\left\{M_{1 \mathrm{~B}}, M_{2 \mathrm{~B}}\right\} & \text { for odd } n .
\end{array}
$$

The free energies of the sublattices are of the form

$$
\begin{aligned}
& \left\{F\left(X_{n}, Y_{n}, Z_{n}\right)\right\} \rightarrow \\
& \left\{F\left(X_{n}^{\mathrm{A}}, Y_{n}^{\mathrm{A}}, Z_{n}^{\mathrm{A}}\right)\right\} \quad \text { for even } n, \\
& \left\{F\left(X_{n}^{\mathrm{B}}, Y_{n}^{\mathrm{B}}, Z_{n}^{\mathrm{B}}\right)\right\} \quad \text { for odd } n \text {, }
\end{aligned}
$$

and then the specific heat is to be defined accordingly, that is

$$
\begin{aligned}
& \left\{C\left(X_{n}, Y_{n}, Z_{n}\right)\right\} \rightarrow \\
& \left\{C\left(X_{n}^{\mathrm{A}}, Y_{n}^{\mathrm{A}}, Z_{n}^{\mathrm{A}}\right)\right\} \quad \text { for even } n, \\
& \left\{C\left(X_{n}^{\mathrm{B}}, Y_{n}^{\mathrm{B}}, Z_{n}^{\mathrm{B}}\right)\right\} \quad \text { for odd } n \text {, }
\end{aligned}
$$

and finally the susceptibility is given as

$$
\begin{aligned}
& \left\{\chi\left(X_{n}, Y_{n}, Z_{n}\right)\right\} \rightarrow \\
& \left\{\chi_{1}\left(X_{n}^{\mathrm{A}}, Y_{n}^{\mathrm{A}}, Z_{n}^{\mathrm{A}}\right)\right. \\
& \left.+\chi_{2}\left(X_{n}^{\mathrm{A}}, Y_{n}^{\mathrm{A}}, Z_{n}^{\mathrm{A}}\right)\right\} \\
& \left\{\chi_{1}\left(X_{n}^{\mathrm{B}}, Y_{n}^{\mathrm{B}}, Z_{n}^{\mathrm{B}}\right)\right. \\
& \left.+\chi_{2}\left(X_{n}^{\mathrm{B}}, Y_{n}^{\mathrm{B}}, Z_{n}^{\mathrm{B}}\right)\right\}
\end{aligned}
$$

Let us note also that in order to use as a guide in calculating the temperature dependent PDs, first we obtain the GS PDs on the $\left(J_{2} /\left|J_{1}\right|, J_{3} / q\left|J_{1}\right|\right)$ planes for given $H / q\left|J_{1}\right|$, i.e. $H_{1}=H_{2}=H$, and on the $\left(H / q\left|J_{1}\right|, J_{3} / q\left|J_{1}\right|\right)$ for given $J_{2} /\left|J_{1}\right|$. The GS energies may be obtained from Eq. (1) in units of $J_{1}$ by rewriting it as

$$
\begin{aligned}
& \frac{E}{q\left|J_{1}\right|}=-\sum_{\langle\text {plaq }\rangle}\left[\frac{J_{1}}{\left|J_{1}\right|} S_{i} S_{j}+\frac{J_{2}}{\left|J_{1}\right|} \sigma_{i^{\prime}} \sigma_{j^{\prime}}\right. \\
& +\frac{J_{3}}{q\left|J_{1}\right|}\left(S_{i} \sigma_{i^{\prime}}+S_{j} \sigma_{j^{\prime}}\right) \\
& \left.+\frac{H}{q\left|J_{1}\right|}\left(S_{i}+S_{j}+\sigma_{i^{\prime}}+\sigma_{j^{\prime}}\right)\right],
\end{aligned}
$$

\begin{tabular}{|c|c|c|c|c|c|}
\hline I & $\begin{array}{l}+1 / 2+1 / 2 \\
+1 / 2 \\
+1 / 2\end{array}$ & II & $\begin{array}{ll} \pm 1 / 2 & \mp 1 / 2 \\
\mp 1 / 2 & \pm 1 / 2\end{array}$ & III & $\begin{array}{ll}+1 / 2 & +1 / 2 \\
\mp 1 / 2 & \pm 1 / 2\end{array}$ \\
\hline IV & $\begin{array}{ll} \pm 1 / 2 & \mp 1 / 2 \\
+1 / 2 & +1 / 2\end{array}$ & V & $\begin{array}{ll}\mp 1 / 2 & \pm 1 / 2 \\
\mp 1 / 2 & \pm 1 / 2\end{array}$ & & \\
\hline
\end{tabular}

where the summation is over all the central plaquette and each plaquette consists of four NN pairs of the two-layer system with one pair $\langle i j\rangle$ from layer $G_{1}$, one pair $\left\langle i^{\prime} j^{\prime}\right\rangle$ from layer $G_{2}$, and two pairs $\left\langle i i^{\prime}\right\rangle$ and $\left\langle j j^{\prime}\right\rangle$ connecting layers $G_{1}$ and $G_{2}$ between the adjacent spins only. The
GS PDs are obtained by comparing the values of the energy for different spin configurations and then the GS configurations are the ones with the lowest energy for given values of the system parameters. Let us note also that the coordination number $q$ is hidden in the GS energy equations, therefore, the GS PDs are obtained for general $q$ values. As a result, we have obtained the following five different types of GS configurations as given in Table.

TABLE

The GS configurations.

While the phases I and II are the FM and AFM phases, the phases III and IV are the surface FM phases with layer $G_{2}$ or $G_{1}$ having zero magnetizations separately, respectively. The phase $\mathrm{V}$ is the mixed phase, i.e. the interlayer interaction is FM type but the intralayer interactions are AFM type.

The nature of the phase transitions is determined from the thermal behaviors of the magnetizations, free energy and response functions, thus the next section is devoted to the illustrations and discussions of the GS and temperature dependent PDs of the model.

\section{Ground state and temperature dependent phase diagrams}

In this section we first illustrate the distinct GS phase diagrams and then using the thermal variations of magnetizations, response functions and free energy together with the guidance of the GS PDs the distinct temperature dependent PDs are obtained and exhibited. The GS PDs are obtained for general $q$ and where the multiphase lines separate the GS configurations as given in Table. The temperature dependent PDs are obtained according to GS PDs by taking horizontal or perpendicular lines cutting through the different GS configurations. They are obtained for $q=3,4$ and 6 and their phase transition lines are labeled with gray solid, dashed and black solid lines, respectively. The arrows indicate the phase separation points according to the GS PDs. Meanwhile, we only consider the positive external magnetic fields since we know that $M(-H)=-M(H)$.

The first GS PD is obtained on the $\left(J_{2} /\left|J_{1}\right|, J_{3} / q\left|J_{1}\right|\right)$ plane for $H / q\left|J_{1}\right|=0.5$, see Fig. 2a, where the phases (V, I), (II, IV, I), (II, IV) and (II, V) are separated with the multiphase lines. The temperature dependent PDs are obtained accordingly for $H / q\left|J_{1}\right|=0.5$ on the $\left(J_{3} /\left|J_{1}\right|, k T /\left|J_{1}\right|\right)$ planes for $J_{2} /\left|J_{1}\right|=-4.0$ and -0.5 which cuts through the phases (II, V) and (II, IV, I) and on the $\left(J_{2} /\left|J_{1}\right|, k T /\left|J_{1}\right|\right)$ planes for $J_{3} / q\left|J_{1}\right|=2.0$ and 

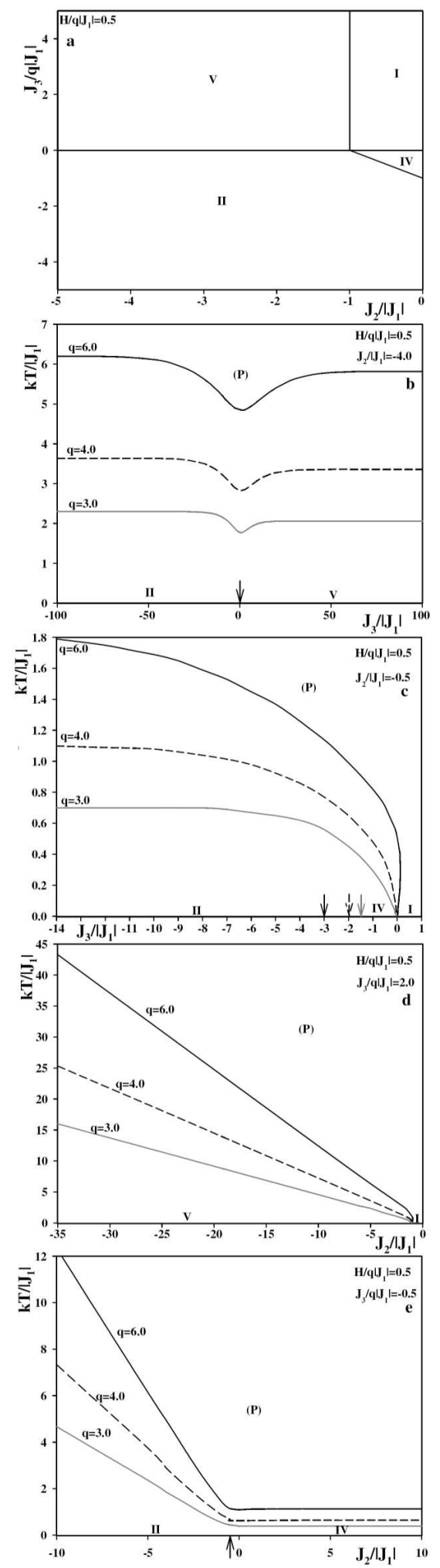

Fig. 2. (a) The GS PD on the $\left(J_{2} /\left|J_{1} /, J_{3} / q\right| J_{1} \mid\right)$ planes for $H / q\left|J_{1}\right|=0.5$. The temperature dependent PDs on the $\left(J_{3} /\left|J_{1}\right|, k T /\left|J_{1}\right|\right)$ planes for $H / q\left|J_{1}\right|=0.5$ with $q=3,4,6$; (b) $J_{2} /\left|J_{1}\right|=-4.0$ and (c) $J_{2} /\left|J_{1}\right|=$ -0.5 , and on the $\left(J_{2} /\left|J_{1}\right|, k T /\left|J_{1}\right|\right)$ planes for $H / q\left|J_{1}\right|=$ 0.5 with $q=3,4$ and 6 ; (d) $J_{3} / q\left|J_{1}\right|=2.0$ and (e) $J_{3} / q\left|J_{1}\right|=-0.5$.
-0.5 which cuts through the phases $(\mathrm{V}, \mathrm{I})$ and (II, IV) and which are displayed in Figs. $2 \mathrm{~b}-\mathrm{e}$, respectively. In Fig. $2 \mathrm{~b}$, the $T_{\mathrm{N}}$-lines start from constant temperatures at higher negative values of $J_{3} /\left|J_{1}\right|$ and which are seen at higher $k T /\left|J_{1}\right|$ with higher $q$. As $J_{3} /\left|J_{1}\right|$ increases, they reach their minima around $J_{3} /\left|J_{1}\right|=0$ and as it increases further they again become constant at some temperatures at higher positive $J_{3} /\left|J_{1}\right|$. The left wings are seen at higher temperature than the right ones, since $J_{1}<0, J_{2}<0$ and $J_{3}<0$ all favors the AFM phase II but while $J_{1}<0$ and $J_{2}<0$ favor AFM phases, $J_{3}>0$ favor FM phase, so phase II is more resistive to temperature than the phase $\mathrm{V}$. The $T_{\mathrm{N}}$-lines do not terminate since both phases II and V are AFM type. Figure 2c shows that the $T_{\mathrm{N}}$-lines start from constant temperatures at higher negative values of $J_{3} /\left|J_{1}\right|$ and as $J_{3} /\left|J_{1}\right|$ increases their temperatures decrease and all go to zero temperature at zero $J_{3} /\left|J_{1}\right|$. The reentrant behavior is exhibited by the $T_{\mathrm{N}}$-line for $q=6$ because of the existence of two $T_{\mathrm{N}}$ 's. The $T_{\mathrm{N}}$-lines terminate in the phase region $\mathrm{I}$, since for the FM phases when there is external magnetic field acting on the layers no second-order phase transitions are seen. Figure $2 \mathrm{~d}$ is similar to Fig. $2 \mathrm{c}$, but now the $T_{\mathrm{N}}$-lines increase towards higher temperatures as $J_{2} /\left|J_{1}\right|$ becomes more negative and all the $T_{\mathrm{N}}$-lines go to zero temperature before $J_{2} /\left|J_{1}\right|=0$. Again the reentrant behavior is seen for $q=6$. The left hand side of Fig. 2e is similar to Fig. 2d, but now the $T_{\mathrm{N}}$-lines cannot go to zero. The upper layer is AFM type and the lower layer is FM type for phase IV, thus we always see $T_{\mathrm{N}}$ 's because of the upper layer and the lines of which are at constant temperatures for all positive values of $J_{2} /\left|J_{1}\right|$.

The next GS PD, Fig. 3a, is obtained on the $\left(J_{2} /\left|J_{1}\right|, J_{3} / q\left|J_{1}\right|\right)$ plane for $H / q\left|J_{1}\right|=1.0$, and for which phase III is also seen in addition to other four phases. The temperature dependent PDs may be obtained along the lines cutting through the phases (II, III, V), (II, III, I), (III, I), (V, I), (II, IV) and (II, IV, I). We consider first three cases and present their PDs in Figs. $3 \mathrm{~b}-\mathrm{d}$, respectively. Figure $3 \mathrm{~b}$ is obtained for $J_{2} /\left|J_{1}\right|=-3.5$ on the $\left(J_{3} /\left|J_{1}\right|, k T /\left|J_{1}\right|\right)$ plane. The $T_{\mathrm{N}}$-lines start from constant temperatures at higher negative $J_{3} /\left|J_{1}\right|$ and as $J_{3} /\left|J_{1}\right|$ increases towards zero the $T_{\mathrm{N}}$-lines decrease and they again become constant at some temperatures for positive $J_{3} /\left|J_{1}\right|$. This figure is actually similar to Fig. 2b. Figure 3c is similar to Fig. 2c, but now the $T_{\mathrm{N}}$-lines go to zero temperature at lower positive values of $J_{3} /\left|J_{1}\right|$ for lower $q$. The last PD is obtained on the $\left(J_{2} /\left|J_{1}\right|, k T /\left|J_{1}\right|\right)$ plane for $J_{3} / q\left|J_{1}\right|=0.5$ and it is very similar to Fig. $2 \mathrm{~d}$, but its $T_{\mathrm{N}}$ 's are seen at higher temperatures. Let us note also that the $T_{\mathrm{N}}$-lines exhibit reentrant behavior for $q=6$ in the last two PDs. We have also obtained the temperature dependent PDs for (II, IV, I), (V, I) and (II, IV) phase transitions according to GS PD (Fig. 3a) but we do not illustrate them in here. The first one is obtained on the $\left(J_{3} /\left|J_{1}\right|, k T /\left|J_{1}\right|\right)$ plane for $J_{2} /\left|J_{1}\right|=-0.7$ and is similar to Fig. $2 \mathrm{c}$, but the $T_{\mathrm{N}}$-lines go to zero temperature at 

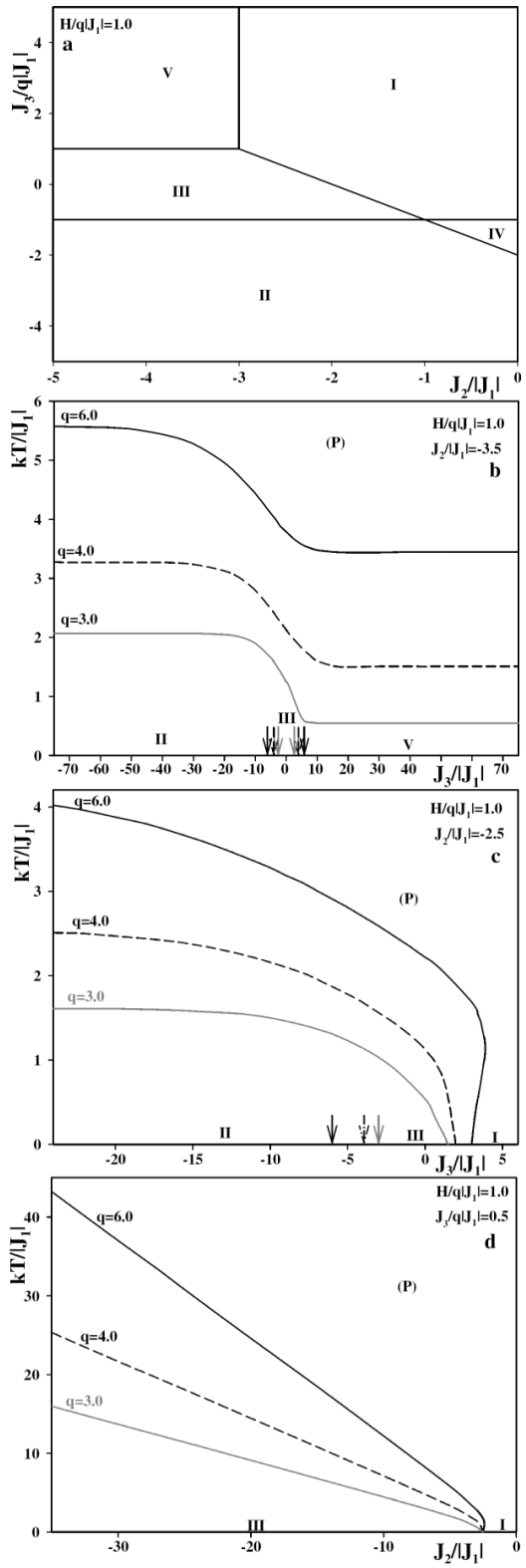

Fig. 3. (a) The GS PD on the $\left(J_{2} /\left|J_{1} /, J_{3} / q\right| J_{1} \mid\right)$ planes for $H / q\left|J_{1}\right|=1.0$. The temperature dependent PDs on the $\left(J_{3} /\left|J_{1}\right|, k T /\left|J_{1}\right|\right)$ planes for $H / q\left|J_{1}\right|=$ 1.0 with $q=3,4$ and 6 ; (b) $J_{2} /\left|J_{1}\right|=-3.5$ and (c) $J_{2} /\left|J_{1}\right|=-2.5$, and (d) on the $\left(J_{2} /\left|J_{1}\right|, k T /\left|J_{1}\right|\right)$ plane for $H / q\left|J_{1}\right|=1.0$ with $q=3,4$ and 6 for $J_{3} / q\left|J_{1}\right|=0.5$.

lower $J_{3} /\left|J_{1}\right|$ for higher $q$. The next two are obtained on the $\left(J_{2} /\left|J_{1}\right|, k T /\left|J_{1}\right|\right)$. First one for $J_{3} / q\left|J_{1}\right|=3.0$ is similar to Fig. $2 \mathrm{~d}$, but the end of the $T_{\mathrm{N}}$-lines moves towards left in comparison. Last one for $J_{3} / q\left|J_{1}\right|=-1.5$ is similar to Fig. 2e, but its $T_{\mathrm{N}}$ 's are higher.

Our next distinct GS PD, Fig. 4a, is obtained on the $\left(H / q\left|J_{1}\right|, J_{3} / q\left|J_{1}\right|\right)$ plane for $J_{2} /\left|J_{1}\right|=-0.5$ and which shows that $(\mathrm{V}, \mathrm{I}),(\mathrm{V}, \mathrm{IV}, \mathrm{I}),(\mathrm{II}, \mathrm{IV}, \mathrm{V}),(\mathrm{II}, \mathrm{IV}, \mathrm{I})$ and (II, V) phase transitions are possible. We have not presented the temperature dependent PDs for the first three
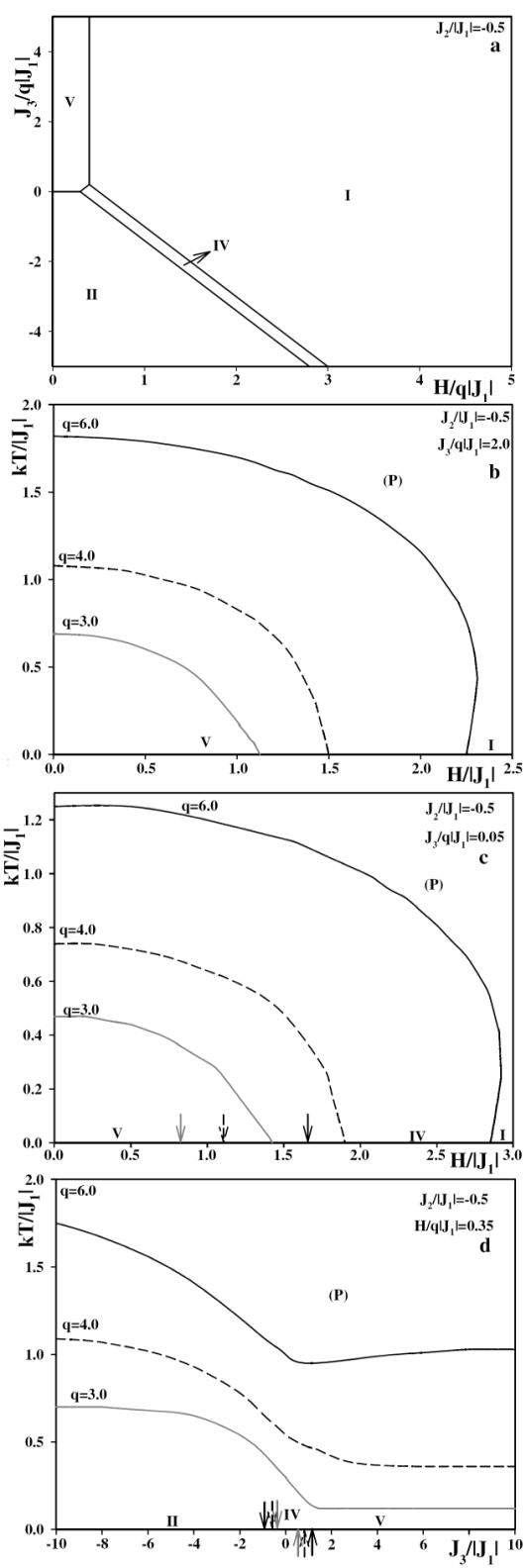

Fig. 4. (a) The GS PD on the $\left(H / q\left|J_{1} /, J_{3} / q\right| J_{1} \mid\right)$ planes for $J_{2} /\left|J_{1}\right|=-0.5$. The temperature dependent PDs on the $\left(H /\left|J_{1}\right|, k T /\left|J_{1}\right|\right)$ planes for $J_{2} /\left|J_{1}\right|=$ -0.5 with $q=3,4$ and 6 ; (b) $J_{3} / q\left|J_{1}\right|=2.0$ and (c) $J_{3} / q\left|J_{1}\right|=0.05$, and (d) on the $\left(J_{3} /\left|J_{1}\right|, k T /\left|J_{1}\right|\right)$ plane for $J_{2} /\left|J_{1}\right|=-0.5$ with $q=3,4$ and 6 for $H / q\left|J_{1}\right|=0.35$.

phase transitions (V, I), (V, IV, I) and (II, IV, V) before. Thus the first two are obtained on the $\left(H /\left|J_{1}\right|, k T /\left|J_{1}\right|\right)$ plane for $J_{3} / q\left|J_{1}\right|=2.0$ and 0.05 and presented in Figs. $4 \mathrm{~b}$ and $\mathrm{c}$, respectively. The $T_{\mathrm{N}}$-lines start from higher temperatures for higher $q$ and as $H /\left|J_{1}\right|$ increases their temperatures decrease and they go to zero temperature at lower $H /\left|J_{1}\right|$ for lower $q$ for both of the PDs. The last one is obtained for $H / q\left|J_{1}\right|=0.35$ on the $\left(J_{3} /\left|J_{1}\right|, k T /\left|J_{1}\right|\right)$ plane. The $T_{\mathrm{N}}$-lines start from higher temperatures for higher $q$ in phase region II and as 

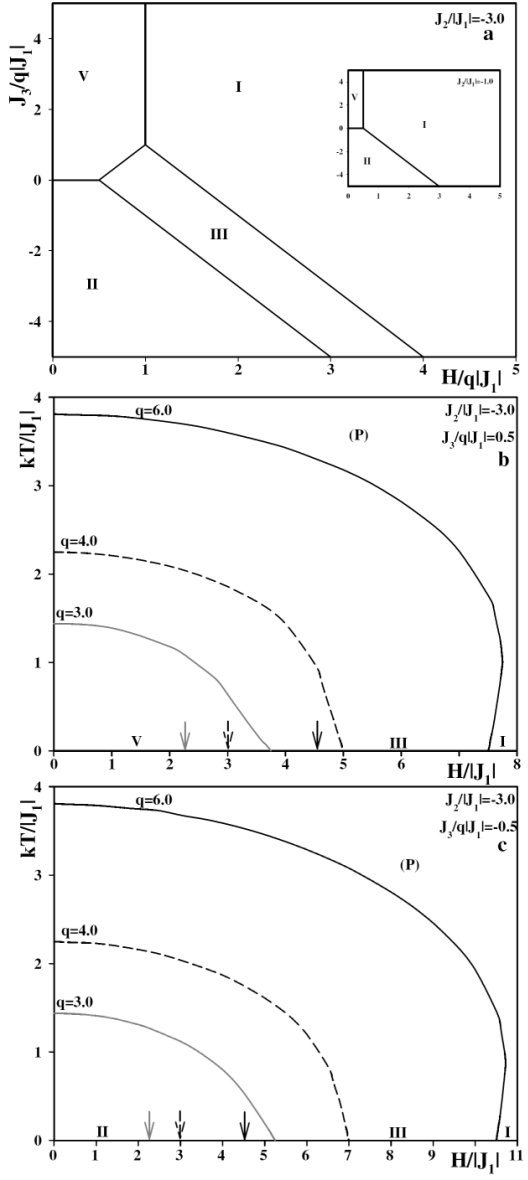

Fig. 5. (a) The GS PDs on the $\left(H / q\left|J_{1} /, J_{3} / q\right| J_{1} \mid\right)$ planes for $J_{2} /\left|J_{1}\right|=-3.0$ and in the inset for $J_{2} /\left|J_{1}\right|=-1.0$. The temperature dependent PDs on the $\left(H /\left|J_{1}\right|, k T /\left|J_{1}\right|\right)$ planes for $J_{2} /\left|J_{1}\right|=-3.0$ with $q=3$, 4 and 6 ; (b) $J_{3} / q\left|J_{1}\right|=0.5$ and (c) $J_{3} / q\left|J_{1}\right|=-0.5$

$J_{3} /\left|J_{1}\right|$ increases their $T_{\mathrm{N}}$ 's decrease until $J_{3} /\left|J_{1}\right| \simeq 0.0$ about phase IV and as $J_{3} /\left|J_{1}\right|$ increases further $T_{\mathrm{N}}$-lines become constant again in phase region V. Since we have already presented the behaviors of the $T_{\mathrm{N}}$-lines for the rest of the transitions, instead of giving their illustrations we just give some comparisons. The first one with (II, IV, I) on the $\left(H /\left|J_{1}\right|, k T /\left|J_{1}\right|\right)$ plane for $J_{3} / q\left|J_{1}\right|=$ -2.0 is similar to Fig. $2 \mathrm{c}$ and its $T_{\mathrm{N}}$-lines end at lower $H /\left|J_{1}\right|$ for lower $q$. The next two are for $H / q\left|J_{1}\right|=0.15$ and 2.0 on the $\left(J_{3} /\left|J_{1}\right|, k T /\left|J_{1}\right|\right)$ plane corresponding to the phase transitions (II, V) and (II, IV, I), respectively. The first one is similar to Fig. $2 \mathrm{~b}$ but its $T_{\mathrm{N}}$-lines are at lower temperatures and the concavities are much deeper. And the last one is similar to Fig. 2c, however the $T_{\mathrm{N}}$-lines go to zero temperature at lower $J_{3} /\left|J_{1}\right|$ for higher $q$.

The last GS PDs, Fig. 5a, are obtained on the $\left(H / q\left|J_{1}\right|, J_{3} / q\left|J_{1}\right|\right)$ planes for $J_{2} /\left|J_{1}\right|=-3.0$ and in the inset $J_{2} /\left|J_{1}\right|=-1.0$. The inset shows that there are only three configurations, i.e. I, II and V, but phase III also lies along the multiphase line separating the phases I and II. In the main figure it is clear that for $J_{2} /\left|J_{1}\right|=-3.0$, the phase III is actually contained in a region separating the phases I and II. Thus, Fig. 5b and c are obtained on the $\left(H /\left|J_{1}\right|, k T /\left|J_{1}\right|\right)$ planes for $J_{3} / q\left|J_{1}\right|=0.5$ and -0.5 , corresponding to the phase transitions (V, III, I) and (II, III, I), respectively. The behaviors of the $T_{\mathrm{N}}$-lines are similar for both cases, i.e. they both start from higher temperatures for higher $q$ for $H /\left|J_{1}\right|=0.0$ and go to zero temperature at lower $H /\left|J_{1}\right|$ for lower $q$. The reentrant behavior is also seen for $q=6$ only. We have also calculated the PDs for the rest of the phase transitions according to Fig. 5a. We have obtained the PD on the $\left(H /\left|J_{1}\right|, k T /\left|J_{1}\right|\right)$ plane for $J_{3} / q\left|J_{1}\right|=3.0$ and found that it only differs with higher $T_{\mathrm{N}}$ values in comparison with Fig. 4b. The last PDs that we have obtained on the $\left(J_{3} /\left|J_{1}\right|, k T /\left|J_{1}\right|\right)$ planes for $H / q\left|J_{1}\right|=0.25,0.75$ and 2.0 for the transitions corresponding to (II, V), (II, III, V) and (II, III, I), respectively. The first one is similar to Fig. 2b, but its $T_{\mathrm{N}}$ values are lower. The next one looks alike with Fig. 3 b again with lower $T_{\mathrm{N}}$ temperatures. And the last one is similar to Fig. $3 \mathrm{c}$, but now the $T_{\mathrm{N}}$-lines go to zero temperature at lower $J_{3} /\left|J_{1}\right|$ for higher $q$. In concluding the discussion of the PDs we should say that we have obtained all the possible distinct GS PDs and according to them we have obtained the distinct temperature dependent PDs corresponding to different phase transitions.

\section{A brief summary and conclusion}

As a summary we have studied the statistical mechanics of the Ising model on a two-layer Bethe lattice with AFM interactions for each layer and either AFM or FM interaction between the layers by using the pairwise approach for given values of coordination number $q$. The order-parameters, response functions and free energy are obtained in terms of the exact recursion relations in a pairwise approach. In the guidance of the GS PDs, the temperature dependent PDs of the model are also studied in detail for given coordination numbers $q=3,4$ and 6 . It was found that the system presents only second-order phase transitions with different thermal behaviors for all values of $q$. In addition, two Néel temperatures, $T_{\mathrm{N}}$, are found for $q=6$ only.

Our found results display all the general characteristics of the spin- $1 / 2$ Ising model as given in detail in the previous section. To end this we mention that the model with AFM intralayer and with AFM or FM interlayer has not been studied, thus no comparison is possible.

\section{Acknowledgments}

This work is supported by the Scientific and Technological Research Council of Turkey (TUBITAK) with fund No. 107 T358. 


\section{References}

[1] Z.B. Li, Z. Shuai, Q. Wang, H.J. Luo, L. Schülke, J. Phys. A, Math. Gen. 34, 6069 (2001).

[2] A.M. Ferrenberg, D.P. Landau, J. Appl. Phys. 70, 6215 (1991)

[3] M.L. Lyra, C.R. da Silva, Phys. Rev. B 47, 526 (1993).

[4] J. Oitmaa, I.G. Enting, J. Phys. A, Math. Gen. 8, 1097 (1975)

[5] A. Lipowski, M. Suzuki, Physica A 198, 227 (1993).

[6] A. Lipowski, Physica A 250, 373 (1998).

[7] T. Horiguchi, A. Lipowski, N. Tsushima, Physica A 224, 626 (1996).

[8] L. Bahmad, A. Benyoussef, H. Ez-Zahraouy, J. Magn. Magn. Mater. 238, 115 (2002).

[9] Z. Weihong, Phys. Rev. B 59, 387 (1999).

[10] J. Wosiek, Phys. Rev. B 49, 15023 (1994).

[11] S.H. Tsai, D.P. Landau, T.C. Schulthess, J. Appl. Phys. 91, 6884 (2002).

[12] J.L. Monroe, Physica A 335, 563 (2004).

[13] K.H. Höglund, A.W. Sandvik, Phys. Rev. Lett. 99, 027205 (2007).

[14] A.W. Sandvik, A.V. Chubukov, S. Sachdev, Phys. Rev. B 51, 16483 (1995).

[15] A.V. Chubukov, D.K. Morr, Phys. Rev. B 52, 3521 (1995).

[16] L. Yin, M. Troyer, S. Chakravarty, Europhys. Lett. 42, 559 (1998)

[17] Z. Weihong, Phys. Rev. B 55, 12267 (1997).

[18] A.W. Sandvik, D.J. Scalapino, Phys. Rev. Lett. 72, 2777 (1994)
[19] Y.M. Seidov, G.R. Shaulov, J. Phys., Condens. Matter 6, 9621 (1994).

[20] F. Dujardin, B. Stebe, A. Ainane, M. Saber, Chinese J. Phys. 37, 479 (1999).

[21] G. Wiatrowski, G. Bayreuther, K. Pruegl, J. Magn. Magn. Mater. 196-197, 26 (1999).

[22] M.B. Taylor, B.L. Gyorffy, J. Phys., Condens. Matter 3, 4971 (1991).

[23] A. Khater, M.A. Ghantous, M. Fresnau, J. Phys. D, Appl. Phys. 35, 951 (2002).

[24] L. Trallori, P. Politi, A. Rettori, M.G. Pini, J. Phys., Condens. Matter 7, 7561 (1995).

[25] D.P. Landau, K. Binder, Phys. Rev. B 41, 4786 (1990)

[26] L. Peliti, M. Saber, Physica A 262, 505 (1999).

[27] H. Ez-Zahraouy, L. Bahmad, A. Benyoussef, Physica $A$ 358, 86 (2005).

[28] M. Bengrine, A. Benyoussef, H. Ez-Zahraouy, F. Mhirech, Physica A 268, 149 (1999).

[29] B. Laaboudi, M. Saber, M. Kerouad, Phys. Status Solidi B 212, 153 (1999).

[30] T. Bouziane, Phys. Status Solidi B 217, 951 (2000).

[31] A. Benyoussef, H. Ez-Zahraouy, J. Phys. I (France) 4, 393 (1994)

[32] C.-K. Hu, N.Sh. Izmailian, K.B. Oganesyan, Phys. Rev. E 59, 6489 (1999).

[33] L. Hao, Z.R. Yang, J. Phys. C, Solid State Phys. 20, L355 (1987).

[34] J.L. Monroe, Phys. Rev. E 71, 017105 (2005). 